\title{
Can palpation-induced muscle pain pattern contribute to the differential diagnosis among temporomandibular disorders, primary headaches phenotypes and possible bruxism?
}

\author{
Yuri-Martins Costa ${ }^{1,2}$, André-Luís Porporatti ${ }^{1}$, Patrícia-dos-Santos Calderon ${ }^{3}$, Paulo-César-Rodrigues \\ Conti $^{1}$, Leonardo-Rigoldi Bonjardim ${ }^{4}$ \\ ${ }^{1}$ Department of Prosthodontics, Bauru School of Dentistry, University of São Paulo, Bauru, Brazil \\ ${ }^{2}$ Section of Orofacial Pain and Jaw Function, Department of Dentistry, Aarhus University, Aarhus, Denmark \\ ${ }^{3}$ Department of Prosthodontics, Federal University of Rio Grande do Norte, Natal, Brazil \\ ${ }^{4}$ Department of Biological Sciences, Bauru School of Dentistry, University of São Paulo, Bauru, Brazil
}

Correspondence:

Section of Orofacial Pain and Jaw Function

Department of Dentistry

Aarhus University

Vennelyst Boulevard, 9

8000 Aarhus $C$

Aarhus, Denmark

yurimartinscosta@yahoo.com.br

\begin{abstract}
Costa YM, Porporatti AL, Calderon PS, Conti PCR, Bonjardim LR. Can palpation-induced muscle pain pattern contribute to the differential diagnosis among temporomandibular disorders, primary headaches phenotypes and possible bruxism?. Med Oral Patol Oral Cir Bucal. 2016 Jan $1 ; 21(1): \mathrm{e} 59-65$.
\end{abstract}

Received: $15 / 04 / 2015$

Accepted: 07/08/2015

http://www.medicinaoral.com/medoralfree01/v21i1/medoralv21ilp59.pdf

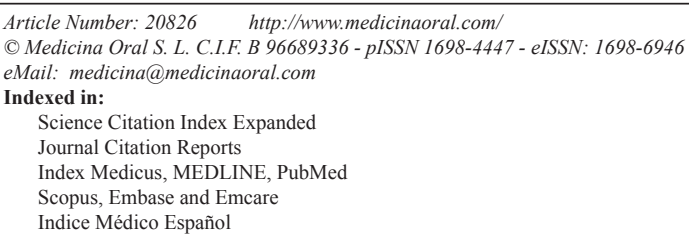

\begin{abstract}
Background: The evaluation of possible differences in the distribution or characteristics of palpation-induced pain in the masticatory muscles could be valuable in terms of diagnostic assessment. The aim of this study was to evaluate the impact of different combinations of anterior temporalis (AT) and masseter palpation-induced pain in the diagnostic of temporomandibular disorder (TMD), primary headaches and bruxism.

Material and Methods: A total of 1200 dental records of orofacial pain adult patients were analyzed. The outcomes were dichotomously classified (presence/absence) as following: a) AT and/or masseter palpation-induced pain; b) myogenous TMD; c) temporomandibular joint (TMJ) arthralgia (arthrogenous TMD); d) migraine; e) tension-type headache (TTH); f) self-reported bruxism. Binomial logistic regression model $(\alpha=5 \%)$ was applied to the data considering the palpation-induced muscle pain as the dependent variable.

Results: Mean age (SD) were 35.7 years (13.4) for 635 included dental records (83\% females). Myogenous and arthrogenous TMD, migraine, TTH and bruxism were mainly associated with, respectively, masseter palpationinduced pain $(p<0.001-\mathrm{OR}=5.77,95 \% \mathrm{CI} 3.86-8.62)$, AT or masseter palpation-induced pain $(p<0.001-\mathrm{OR}=2.39$, 95\% CI 1.57-3.63), bilateral AT palpation-induced pain $(p<0.001-\mathrm{OR}=2.67,95 \% \mathrm{CI} 1.64-4.32)$, masseter and AT palpation-induced pain $(p=0.009$ - $\mathrm{OR}=1.62,95 \% \mathrm{CI} 1.12-2.33)$ and bilateral masseter palpation-induced pain $(p=0.01-\mathrm{OR}=1.74,95 \%$ CI $1.13-2.69)$.
\end{abstract}


Conclusions: Palpation-induced pain in the masticatory muscles may play a role in the differential diagnosis among painful TMD, primary headaches and bruxism.

Key-words: Diagnosis, temporomandibular joint disorders, migraine, tension-type headache, bruxism.

\section{Introduction}

The pain assessment is a challenge task, considering that pain is a subjective experience and it is determined by a complex system of neuronal connections and it is influenced by biological, emotional and behavioral factors (1). In this scenario, the palpation-induced pain is an easy and informative psychophysical technique for the clinical assessment of deep pain (2). In particular, the diagnostic classification of painful musculoskeletal conditions, e.g., temporomandibular disorder (TMD), defined as a collection of clinical problems that involve the masticatory muscles, the temporomandibular joint (TMJ) and associated structures, are mostly based on the information of pain upon palpation of the muscles and joint (1). Furthermore, palpation-induced pain could be considered relevant for the assessment of primary headaches disorders and, in fact, it is useful to differentiate sub-types of tension-type headache (TTH), i.e., with or without pericranial tenderness (3). Finally, bruxism, a non-painful disorder, which could be defined as a sleep or wakefulness repetitive jaw-muscle activity characterized by clenching or grinding of the teeth and/or by bracing or thrusting of the mandible (4), is often associated with muscle pain and fatigue and it is considered a risk factor for TMD (5).

Researches focusing the muscle pain upon palpation or more standardized and reliable techniques, e.g., pressure pain threshold (PPT), have been elucidating some underlying mechanisms of myofascial pain and primary headaches, i.e., peripheral and central sensitization (6-8). Also, experimental tooth clenching models have unraveled the relationship between repetitive muscle activity and pain (9). However, in the clinical settings there is a lack of evidence regarding the pattern of palpation-induced muscle pain in the aforementioned disorders. Furthermore, considering the high prevalence of primary headaches, TMD, and bruxism (10-12) and their presumed triple comorbidity (13), the evaluation of possible differences in the distribution or characteristics of palpation-induced pain in the masticatory muscles could be valuable in terms of diagnostic assessment.

Based on that, the objective of this study was to evaluate the impact of different combinations of anterior temporalis (AT) and masseter palpation-induced pain in the diagnostic of temporomandibular disorders, primary headaches phenotypes and possible bruxism.

\section{Material and Methods \\ - Design and Sample}

This was a cross-sectional study with a retrospective design conducted in Brazil and performed in accordance with the ethical standards of the Declaration of Helsinki and its later amendments and approved by Ethic Committee of Human Research of Bauru School of Dentistry, University of Sao Paulo.

The sample size comprised all the population (1200 dental records) of patients who lived in the metropoli$\tan$ region of Bauru and were referred or sought care for orofacial pain at the Orofacial Pain Clinic of Bauru School of Dentistry, a tertiary care center, among 1996 and 2009. Qualified professionals in training and under the supervision of two experienced professors and TMD specialists examined all the patients. The data collection occurred between 2010 and 2011. All subjects who took part in the study gave their informed consent.

All records comprised details of the clinical examination which consisted of comprehensive medical history, information about chief pain complaint, headache, intensity, frequency and quality of pain/headache, history of trauma, parafunctional habits, i.e., awake or sleep bruxism (self-report), medications intake and presence of systematic or degenerative diseases. The physical examination comprised the measurements of mandibular movements (open, lateral and protrusive) and assessment of TMJ noises and manual palpation of temporomandibular joint (TMJ), masticatory muscles and also sternocleidomastoid and trapezius.

According to the baseline clinical exam, the diagnostic algorithm of myogenous TMD (myalgia and myofascial pain) and TMJ arthralgia (arthrogenous TMD) were made according the guidelines of the American Academy of Orofacial Pain (14) whilst the diagnostic of TTH or migraine were made according the International Classification of Headache Disorders (ICHD 2) (15). Also, the diagnostic of possible bruxism was defined according the proposals for a grading diagnostic system recently published by a group of experts, that suggest the term "possible" when only anamnestic approach is accomplished (4).

The inclusion criteria were: a) adults aged 18 years or more; b) a clearly description of the assessment of AT and masseter palpation-induced pain; c) the address of parafunctional habits (sleep or awake clenching/grinding) by self-report. 
The exclusion criteria were: a) presence of neurological or neuropathic diseases; b) fibromyalgia; c) systemic arthritis; d) other primary headaches than migraine or tensiontype headache; e) history of intracranial disorders, vascular disorders and other major causes of headache than temporomandibular joint disorders, listed in the ICHD 2 $(15)$, f) lack of information which avoided the diagnostic of temporomandibular disorders (TMDs), migraine and TTH. Therefore, we dealt with the missing data by excluding incomplete records.

- Variables

Based on the clinical examination and eligibility criteria, the variables of this report were retrospectively identified and dichotomously classified as following: a) presence/absence of AT and/or masseter pain to palpation; b) presence/absence of myogenous TMD; c) presence/absence of TMJ arthralgia; d) presence/absence of migraine; e) presence/absence of TTH; f) presence/ absence of possible bruxism.

- Statistics

The description of age and the distribution of gender and diagnostic of myogenous TMD, TMJ arthralgia, migraine, TTH and possible bruxism were made for each group.

The inferential analysis was made through binomial logistic regression model stepwise backward considering the following dependent variables: a) AT palpation- induced pain; b) unilateral AT palpation-induced pain; c) bilateral AT palpation-induced pain; d) masseter palpation-induced pain; e) unilateral masseter palpationinduced pain; f) bilateral masseter palpation-induced pain; g) AT or masseter palpation-induced pain; h) AT and masseter palpation-induced pain; i) only AT palpation-induced pain; j) only masseter palpation-induced pain. The independent variables in all analyses were the diagnostic of (1) myogenous TMD, (2) TMJ arthralgia, (3) migraine, (4) TTH and (5) possible bruxism. We considered a $5 \%$ of significance level and $95 \%$ of Confidence Interval (CI). The Statistical Package for the Social Sciences (SPSS) v.18.0 (IBM Corp., USA) was used to perform the tests.

\section{Results}

We analyzed 1200 dental records of which 635 fulfilled the criteria and were selected. The main reason for exclusion was the lack of information, which prevented the diagnostic of the target conditions. Demographic characteristics and diagnostic categories were: $83 \%$ were women, the mean age (SD) was 35.7 (13.4) years and $74 \%$ had myogenous TMD, 59\% TMJ arthralgia, $28 \% \mathrm{TTH}, 14 \%$ migraine and $80 \%$ possible bruxism (Fig. 1).

Tables 1-4 show the results of the logistic regression

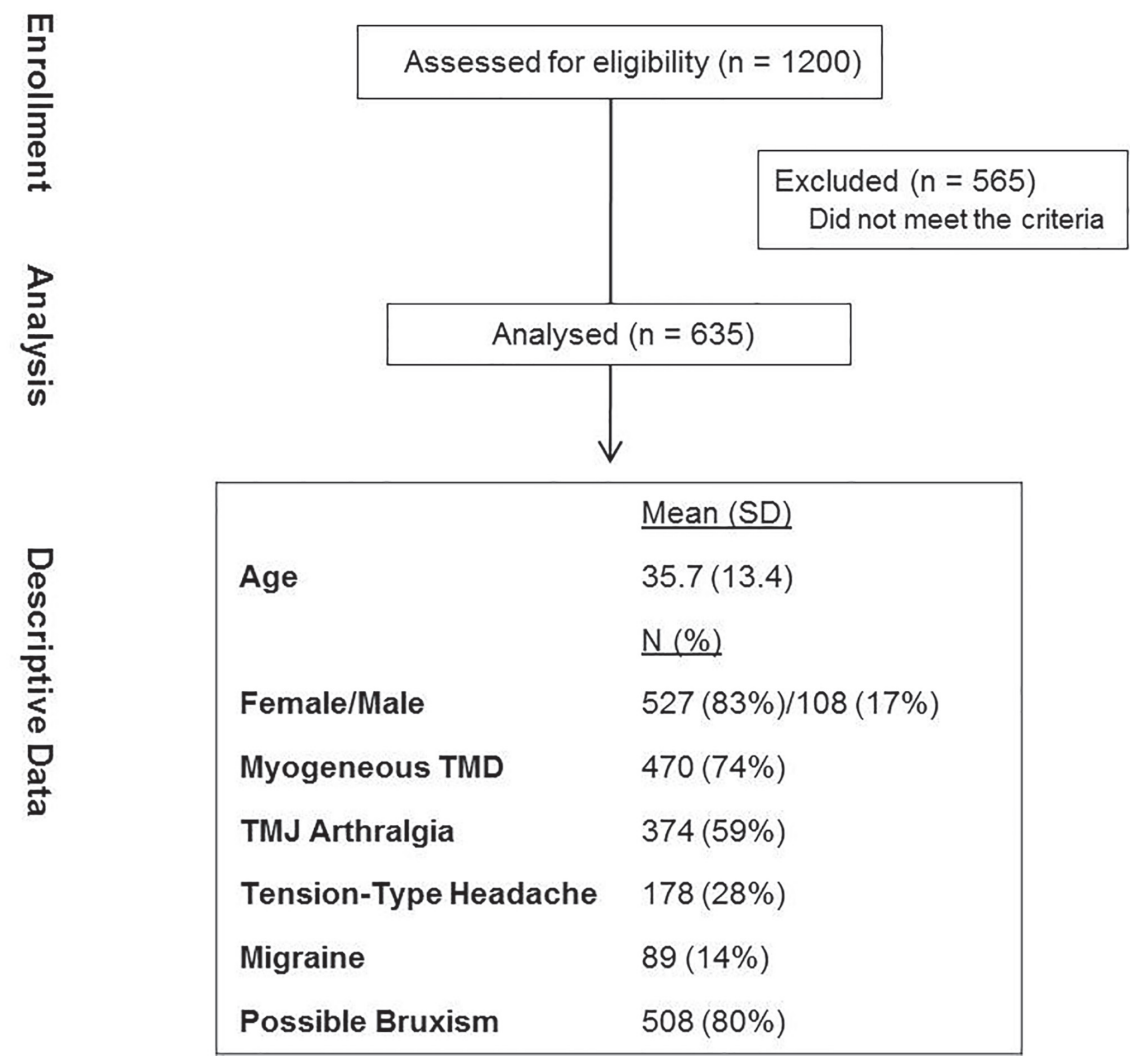

Fig. 1. Flowdiagram and descriptive data of the study. 
analysis. After 1 step, only migraine did not make a significant contribution considering AT palpation-induced pain $(p>0.05)$, whereas for masseter palpation-induced pain, only myogenous TMD $(p<0.001-\mathrm{OR}=5.77,95 \%$ $\mathrm{CI}=3.86-8.62)$ and TMJ arthralgia $(p=0.002-\mathrm{OR}=1.79$, $95 \% \mathrm{CI}=1.23-2.59)$ were significantly associated after 3 steps (Table 1).

In relation to unilateral AT palpation-induced pain as the dependent variable, migraine $(p=0.02-\mathrm{OR}=0.42,95 \%$
$\mathrm{CI}=0.2-0.87)$ and $\mathrm{TMJ}$ arthralgia $(p=0.01-\mathrm{OR}=1.69$, $95 \% \mathrm{CI}=1.11-2.59)$ made a significant contribution to the model after 3 steps, whereas for unilateral masseter palpation-induced pain, only myogenous TMD $(p<0.001$ $-\mathrm{OR}=3.12,95 \% \mathrm{CI}=1.89-5.14)$ was significantly associated after 3 steps (Table 2).

Myogenous TMD $(p<0.001-\mathrm{OR}=3.98,95 \% \mathrm{CI}=2.44$ $6.49)$, migraine $(p<0.001-\mathrm{OR}=1.75,95 \% \mathrm{CI}=1.23-2.49)$ and TTH $(p=0.01-\mathrm{OR}=1.58,95 \% \mathrm{CI}=1.09-2.3)$ were

Table 1. Logistic regression analysis. Anterior temporalis (AT) and masseter pain-induced palpation are the dependent variables and myogenous TMD temporomandibular joint (TMJ) arthralgia, migraine, tension-type headache (TTH) phenotypes and possible bruxism are the independent variables.

\begin{tabular}{|c|c|c|c|c|c|c|c|c|}
\hline & \multicolumn{8}{|c|}{ AT pain-induced palpation* } \\
\hline & \multicolumn{8}{|c|}{ Masseter pain-induced palpation } \\
\hline & B & S.E & Wald & $P$ - value & $\mathrm{OR}$ & $95 \%$ & $\mathrm{r} \mathrm{OR}$ & $\begin{array}{c}\text { Cox \& } \\
\text { Snell R }\end{array}$ \\
\hline \multirow[t]{2}{*}{ Myogenous TMD } & 1.15 & 0.2 & 30.57 & $<0.001$ & 3.16 & 2.1 & 4.75 & \multirow[t]{6}{*}{0.11} \\
\hline & 1.75 & 0.2 & 73.51 & $<0.001$ & 5.77 & 3.86 & 8.62 & \\
\hline \multirow[t]{2}{*}{ TMJ Arthralgia } & 0.57 & 0.17 & 10.7 & 0.001 & 1.7 & 1.26 & 2.51 & \\
\hline & 0.58 & 0.18 & 9.45 & 0.002 & 1.79 & 1.23 & 2.59 & \\
\hline \multirow[t]{2}{*}{ Migraine phenotype } & - & - & - & - & - & - & - & \\
\hline & - & - & - & - & - & - & - & \\
\hline \multirow[t]{2}{*}{ TTH phenotype } & 0.54 & 0.19 & 7.92 & 0.004 & 1.7 & 1.17 & 2.5 & \multirow[t]{6}{*}{0.15} \\
\hline & - & - & - & - & - & - & - & \\
\hline \multirow[t]{2}{*}{ Possible Bruxism } & 0.52 & 0.21 & 6.16 & 0.01 & 1.6 & 1.11 & 2.57 & \\
\hline & - & - & - & - & - & - & - & \\
\hline \multirow[t]{2}{*}{ Constant } & -1.16 & 0.2 & 40.76 & $<0.001$ & 0.18 & & & \\
\hline & -0.85 & 0.18 & 22.03 & $<0.001$ & 0.42 & & & \\
\hline
\end{tabular}

* The darker rows indicate the values for AT pain-induced palpation. The lighter rows indicate the values for masseter paininduced palpation.

Table 2. Logistic regression analysis. Unilateral anterior temporalis (AT) and masseter pain-induced palpation are the dependent variables and myogenous TMD, temporomandibular joint (TMJ) arthralgia, migraine, tension-type headache (TTH) phenotypes and possible bruxism are the independent variables.

\begin{tabular}{c}
\hline Unilateral AT pain-induced palpation * \\
Unilateral masseter pain-induced palpation \\
\hline
\end{tabular}

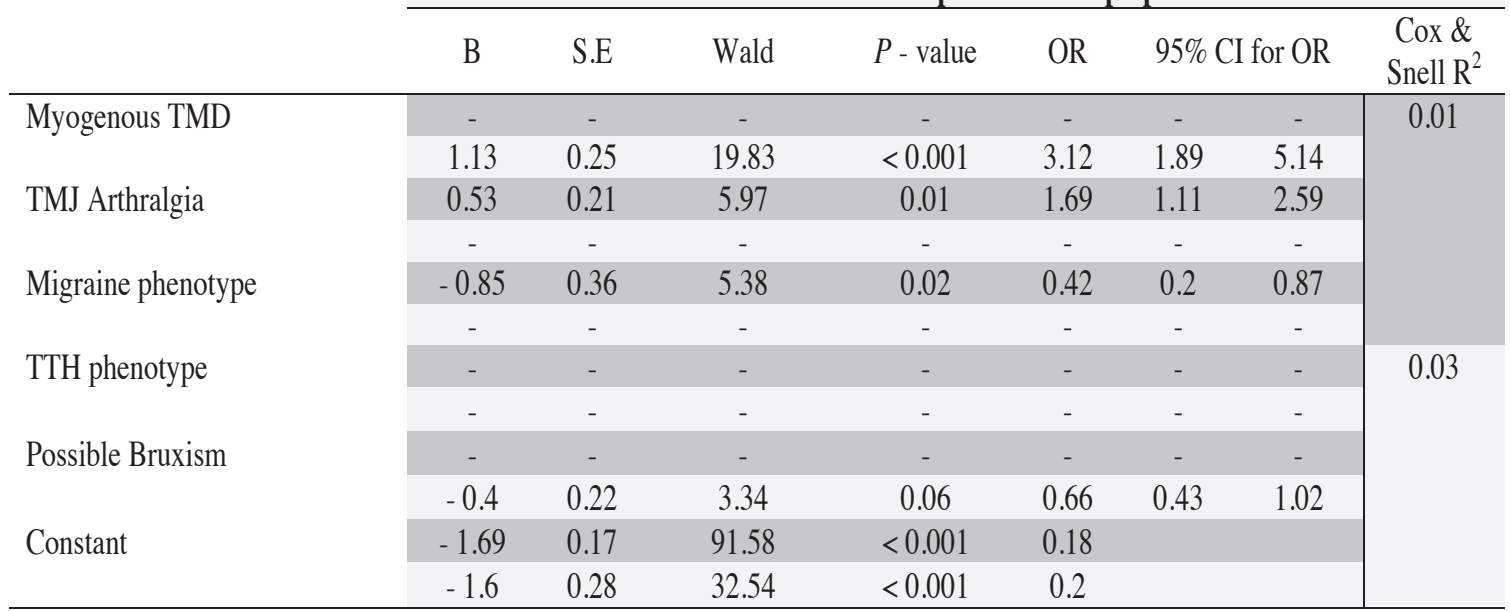

* The darker rows indicate the values for unilateral AT pain-induced palpation. The lighter rows indicate the values for unilateral masseter pain-induced palpation. 
significantly associated with bilateral AT palpation-induced pain after 2 steps, whereas for bilateral masseter palpation-induced pain, myogenous TMD $(p<0.001$ $\mathrm{OR}=2.43,95 \% \mathrm{CI}=1.58-3.74)$, TMJ arthralgia $(p=0.001$ - $\mathrm{OR}=1.75,95 \% \mathrm{CI}=1.23-2.49)$ and possible bruxism ( $p=0.01-\mathrm{OR}=1.74,95 \% \mathrm{CI}=1.13-2.69)$ made a significant contribution to the model after 1 step (Table 3 ).

Only migraine did not make a significant contribution considering palpation-induced muscle pain (AT and/or masseter) after 1 step. Also, TTH and possible bruxism did not make a significant contribution to the model considering AT or masseter palpation-induced pain (Table 4). Finally, in relation to only AT palpation-induced pain, there was no significant association after 5 steps, whereas for only masseter palpation-induced pain, there was a significant association with myogenous TMD ( $p=0.001-\mathrm{OR}=2.28,95 \% \mathrm{CI}=1.39-3.74)$ after 4 steps.

Table 3. Logistic regression analysis. Bilateral anterior temporalis (AT) and bilateral masseter pain-induced palpation are the dependent variables and myogenous TMD, temporomandibular joint (TMJ) arthralgia, migraine, tension-type headache (TTH) phenotypes and possible bruxism are the independent variables.

\section{Bilateral AT pain-induced palpation * Bilateral masseter pain-induced palpation}

\begin{tabular}{|c|c|c|c|c|c|c|c|c|}
\hline & & & & & & & & \\
\hline & B & S.E & Wald & $P$ - value & OR & $95 \%$ & or OR & $\begin{array}{c}\text { Cox \& } \\
\text { Snell R }\end{array}$ \\
\hline \multirow[t]{2}{*}{ Myogenous TMD } & 1.38 & 0.24 & 30.6 & $<0.001$ & 3.98 & 2.44 & 6.49 & \multirow[t]{6}{*}{0.09} \\
\hline & 0.88 & 0.21 & 16.4 & $<0.001$ & 2.43 & 1.58 & 3.74 & \\
\hline \multirow[t]{2}{*}{ TMJ Arthralgia } & - & - & - & - & - & - & - & \\
\hline & 0.56 & 0.17 & 9.94 & 0.001 & 1.75 & 1.23 & 2.49 & \\
\hline \multirow[t]{2}{*}{ Migraine phenotype } & 0.98 & 0.24 & 15.93 & $<0.001$ & 2.67 & 1.64 & 4.32 & \\
\hline & - & - & - & - & - & - & - & \\
\hline \multirow[t]{2}{*}{ TTH phenotype } & 0.46 & 0.19 & 5.85 & 0.01 & 1.58 & 1.09 & 2.3 & \multirow[t]{6}{*}{0.1} \\
\hline & - & - & - & - & - & - & - & \\
\hline \multirow[t]{2}{*}{ Possible Bruxism } & - & - & - & - & - & - & - & \\
\hline & 0.55 & 0.22 & 6.41 & 0.01 & 1.74 & 1.13 & 2.69 & \\
\hline \multirow[t]{2}{*}{ Constant } & -2.06 & 0.23 & 74.72 & $<0.001$ & 0.12 & & & \\
\hline & -1.97 & 0.27 & 50.18 & $<0.001$ & 0.13 & & & \\
\hline
\end{tabular}

* The darker rows indicate the values for bilateral AT pain-induced palpation. The lighter rows indicate the values for bilateral masseter pain-induced palpation.

Table 4. Logistic regression analysis. Anterior temporalis (AT) and/or masseter pain-induced palpation are the dependent variables and myogenous TMD, temporomandibular joint (TMJ) arthralgia, migraine, tension-type headache (TTH) phenotypes and possible bruxism are the independent variables.

\begin{tabular}{|c|c|c|c|c|c|c|c|c|}
\hline & \multicolumn{8}{|c|}{ AT and masseter pain-induced palpation* } \\
\hline & \multicolumn{8}{|c|}{ AT or masseter pain-induced palpation } \\
\hline & $\mathrm{B}$ & S.E & Wald & $P$ - value & OR & $95 \%$ & or OR & $\begin{array}{c}\text { Cox \& } \\
\text { Snell R }\end{array}$ \\
\hline \multirow[t]{2}{*}{ Myogenous TMD } & 1.19 & 0.22 & 29.12 & $<0.001$ & 3.31 & 2.14 & 5.11 & \multirow[t]{6}{*}{0.1} \\
\hline & 1.93 & 0.21 & 78.85 & $<0.001$ & 6.89 & 4.5 & 10.55 & \\
\hline \multirow[t]{2}{*}{ TMJ Arthralgia } & 0.47 & 0.17 & 7.06 & 0.007 & 1.6 & 1.13 & 2.27 & \\
\hline & 0.87 & 0.21 & 16.64 & $<0.001$ & 2.39 & 1.57 & 3.63 & \\
\hline \multirow[t]{2}{*}{ Migraine phenotype } & - & - & - & - & - & - & - & \\
\hline & - & - & - & - & - & - & - & \\
\hline \multirow[t]{2}{*}{ TTH phenotype } & 0.48 & 0.18 & 6.65 & 0.009 & 1.62 & 1.12 & 2.33 & \multirow[t]{6}{*}{0.2} \\
\hline & 0.46 & 0.25 & 3.27 & 0.07 & 1.59 & 0.96 & 2.63 & \\
\hline \multirow[t]{2}{*}{ Possible Bruxism } & 0.47 & 0.21 & 4.78 & 0.02 & 1.6 & 1.05 & 2.46 & \\
\hline & 0.42 & 0.25 & 2.85 & 0.09 & 1.53 & 0.93 & 2.5 & \\
\hline \multirow[t]{2}{*}{ Constant } & -1.96 & 0.27 & 50.04 & $<0.001$ & 0.14 & & & \\
\hline & -1.07 & 0.27 & 15.04 & $<0.001$ & 0.34 & & & \\
\hline
\end{tabular}

* The darker rows indicate the values for AT and masseter pain-induced palpation. The lighter rows indicate the values for AT or masseter pain-induced palpation. 


\section{Discussion}

The main findings of this retrospective study were: a) the greater the number of palpation-induced pain sites, the greater the amount of associated diagnostic categories; b) myogenous TMD was mainly associated with masseter palpation-induced pain; c) TTH was the major primary headache phenotype associated with palpationinduced pain in the masticatory muscles; d) migraine was mainly associated with bilateral AT palpation-induced pain; e) possible bruxism was mainly associated with bilateral masseter palpation-induced pain.

There are evidences supporting the association and the accepted comorbidity between primary headaches and TMD $(11,16)$. Both disorders are clinically related, considering that the prevalence of TMD is higher in headache patients and vice-versa $(17,18)$. Also, a recent study proposed multiple comorbidity among TMD, primary headaches and sleep bruxism (13). Despite this fact and the presumed pathophysiology for these relationships, i.e., common sensory innervation and central nervous system pathways via trigeminal nucleus caudalis, little is known regarding the role of palpation-induced muscle pain in the masticatory muscles on this multiple relationship. On the contrary, sound evidences indicate different patterns of pain distribution between TMD and TTH (19) and masticatory muscle pain sensitivity in TMD, migraine and TTH patients (6-8). Furthermore, this sensitivity is further increased when TMD is associated with headaches (20). Our results are in agreement with the above evidences and suggest different combinations of palpation-induced pain as being related to the diagnostic of painful TMD, primary headaches and bruxism, but also this multiple comorbidity is associated with a greater number of palpation-induced pain sites.

The myogenous TMD is the most common type of painful TMD and it is a disabling disorder (14). In spite the fact of some circularity in the reasoning, since the pain to palpation is a criterion for pain-related TMD (1), it is important to note some differences in the pain distribution. The masseter muscle seems to be important for the diagnostic of myogenous TMD considering that the greatest odds ratio for myogenous TMD were related to the masseter palpation-induced pain. Our results are in line with evidences showing the masseter muscle as the most sensitive site in myofascial TMD pain patients (21). Furthermore, the pain drawings of myofascial TMD pain patients are similar to those of healthy participants under experimental masseter pain (19).

AT palpation-induced pain was associated with primary headache diagnosis. Likewise, despite some circular reasoning, we found different combinations of palpation-induced muscle pain between the primary headaches. Our results suggest that, even it is not completely understood, the nociceptive process in the masticatory muscles seems to play an important role in the TTH underlying mechanisms and the musculoskeletal physical exam could be considered relevant for the diagnostic (22). In fact, the palpation-induced pain is a prominent finding in the TTH population, especially chronic TTH (7). On the other hand, muscle factors are not considered essential in the well-established migraine pathophysiology; however, there are evidences of muscle pain as a presumed consequence of central sensitization process in migraineurs (8). Our results are similar with studies that pointed out masticatory muscle sensitivity in migraine patients, in particular, the bilateral AT sensitivity. We found bilateral AT palpation-induced pain associated with a 2.67 fold increase in odds of having migraine. Also, a possible puzzling aspect is the fact that the mechanical sensitivity reported by migraineurs can be result of the "natural" pericranial tenderness, typical of such population, regardless of the occurrence of migraine attacks. It has been demonstrated that PPT of women with migraine is reduced, even when no myogenous TMD is previously diagnosed (23). As stated before, this could be attributed to the well-known mechanisms of central and peripheral sensitization phenomena. Taken together, the differences in the distribution and patterns of palpation-induced muscle pain could be an important factor when assessing patients with TMD and primary headaches. The high degree of comorbidity among these disorders warrants further research about the role of muscle pain in this complex and often overlooked relationship.

The significant, albeit weak, association between masseter palpation-induced pain and possible bruxism is an interest finding of this study. The majority of evidences on TMD-bruxism relationship support the association between bruxism and symptoms of muscle pain or fatigue (5). Indeed, the report of morning masticatory muscle pain/fatigue is considered a criterion for clinical diagnostic of sleep bruxism (24). Furthermore, epidemiological studies extensively present a positive association between bruxism and painful TMD, especially myogenous TMD (5). However, palpation-induced pain is not sine qua non for myofascial pain; so, it is not possible to presume from the above studies that bruxism is associated with pain to palpation. On the contrary, the association between palpation-induced pain and bruxism was not supported when polysomnographic recordings (PSG) were used as the diagnostic criteria, though one limitation of this study was the small sample size (25). Thus, we propose that our findings support the hypothesis of a possible muscle dysfunction in bruxism patients, which also present higher levels of pain-induced by function and higher muscle activity compared with asymptomatic controls (5). Nevertheless, the clinical value of pain to palpation on the diagnostic of bruxism remains to be established and sound conclusions are not 
possible considering that we used only the self-report for the bruxism assessment with no distinction between sleep and awake bruxism.

This study's strengths were the high degree of representativeness of the orofacial pain population in the clinical setting and the application of regression models for controlling the confounders and the overlap of diagnostics. It is also important to note that we adopted acceptable classification and assessment criteria for TMD, primary headaches and bruxism. On the other hand, the limitations of this study were mostly the lack of a rigorous and standardized protocol for evaluation, considering that there was no possibility of examiner's blinding. Also, the participation of different examiners is also an important limitation. Finally, the cross-sectional characteristic does not allow cause-effect assumptions.

In conclusion, we endorse the importance of the routinely palpation-induced pain examination in the clinical assessment of pain disorders and emphasize its plausible value in terms of differential diagnosis among painful TMD, primary headaches and bruxism, nonetheless this aspect remains to be confirmed and further analyzed in future studies.

\section{References}

1. Schiffman E, Ohrbach R, Truelove E, Look J, Anderson G, Goulet JP, et al. Diagnostic Criteria for Temporomandibular Disorders (DC/ TMD) for Clinical and Research Applications: recommendations of the International RDC/TMD Consortium Network* and Orofacial Pain Special Interest Groupdagger. J Oral Facial Pain Headache. 2014;28:6-27.

2. Futarmal S, Kothari M, Ayesh E, Baad-Hansen L, Svensson P. New palpometer with implications for assessment of deep pain sensitivity. J Dent Res. 2011;90:918-22.

3. Headache Classification Committee of the International Headache Society (IHS). The International Classification of Headache Disorders, 3rd edition (beta version). Cephalalgia. 2013;33:629-808.

4. Lobbezoo F, Ahlberg J, Glaros AG, Kato T, Koyano K, Lavigne GJ, et al. Bruxism defined and graded: an international consensus. J Oral Rehabil. 2013;40:2-4.

5. Manfredini D, Lobbezoo F. Relationship between bruxism and temporomandibular disorders: a systematic review of literature from 1998 to 2008. Oral Surg Oral Med Oral Pathol Oral Radiol Endod. 2010;109:e26-50.

6. Fernandez-de-las-Penas C, Galan-del-Rio F, Fernandez-Carnero J, Pesquera J, Arendt-Nielsen L, Svensson P. Bilateral widespread mechanical pain sensitivity in women with myofascial temporomandibular disorder: evidence of impairment in central nociceptive processing. J Pain. 2009;10:1170-8.

7. Fernandez-de-las-Penas C, Ge HY, Cuadrado ML, Madeleine P, Pareja JA, Arendt-Nielsen L. Bilateral pressure pain sensitivity mapping of the temporalis muscle in chronic tension-type headache. Headache. 2008;48:1067-75.

8. Fernandez-de-las-Penas C, Madeleine P, Cuadrado ML, Ge HY, Arendt-Nielsen L, Pareja JA. Pressure pain sensitivity mapping of the temporalis muscle revealed bilateral pressure hyperalgesia in patients with strictly unilateral migraine. Cephalalgia. 2009;29:670-6. 9. Christensen LV. Jaw muscle fatigue and pains induced by experimental tooth clenching: a review. J Oral Rehabil. 1981;8:27-36.

10. Ciancaglini R, Gherlone EF, Radaelli G. The relationship of bruxism with craniofacial pain and symptoms from the masticatory system in the adult population. J Oral Rehabil. 2001;28:842-8.
11. Goncalves DA, Bigal ME, Jales LC, Camparis CM, Speciali JG. Headache and symptoms of temporomandibular disorder: an epidemiological study. Headache. 2010;50:231-41.

12. Goncalves DA, Dal Fabbro AL, Campos JA, Bigal ME, Speciali JG. Symptoms of temporomandibular disorders in the population: an epidemiological study. J Orofac Pain. 2010;24:270-8.

13. Fernandes G, Franco AL, Goncalves DA, Speciali JG, Bigal ME, Camparis CM. Temporomandibular disorders, sleep bruxism, and primary headaches are mutually associated. J Orofac Pain. 2013;27:14-20

14. Peck CC, Goulet JP, Lobbezoo F, Schiffman EL, Alstergren P, Anderson GC, et al. Expanding the taxonomy of the diagnostic criteria for temporomandibular disorders. J Oral Rehabil. 2014;41:2-23. 15. Headache Classification Subcommittee of the International Headache Society. The International Classification of Headache Disorders: 2nd edition. Cephalalgia. 2004;24 Suppl 1:9-160.

16. Goncalves DA, Camparis CM, Speciali JG, Franco AL, Castanharo SM, Bigal ME. Temporomandibular disorders are differentially associated with headache diagnoses: a controlled study. Clin J Pain. 2011;27:611-5.

17. Ballegaard V, Thede-Schmidt-Hansen P, Svensson P, Jensen R. Are headache and temporomandibular disorders related? A blinded study. Cephalalgia. 2008;28:832-41.

18. Melo CE, Oliveira JL, Jesus AC, Maia ML, de Santana JC, Andrade LS, et al. Temporomandibular disorders dysfunction in headache patients. Med Oral Patol Oral Cir Bucal. 2012;17:e1042-6.

19. Svensson P. Muscle pain in the head: overlap between temporomandibular disorders and tension-type headaches. Curr Opin Neurol. 2007;20:320-5.

20. Anderson GC, John MT, Ohrbach R, Nixdorf DR, Schiffman EL, Truelove ES, et al. Influence of headache frequency on clinical signs and symptoms of TMD in subjects with temple headache and TMD pain. Pain. 2011;152:765-71.

21. Santos Silva RS, Conti PC, Lauris JR, da Silva RO, Pegoraro LF. Pressure pain threshold in the detection of masticatory myofascial pain: an algometer-based study. J Orofac Pain. 2005;19:318-24.

22. Abboud J, Marchand AA, Sorra K, Descarreaux M. Musculoskeletal physical outcome measures in individuals with tensiontype headache: a scoping review. Cephalalgia. 2013;33:1319-36.

23. Sales Pinto LM, de Carvalho JJ, Cunha CO, Dos Santos Silva R, Fiamengui-Filho JF, Rodrigues Conti PC. Influence of myofascial pain on the pressure pain threshold of masticatory muscles in women with migraine. Clin J Pain. 2013;29:362-5.

24. Manfredini D, Ahlberg J, Castroflorio T, Poggio CE, GuardaNardini L, Lobbezoo F. Diagnostic accuracy of portable instrumental devices to measure sleep bruxism: a systematic literature review of polysomnographic studies. J Oral Rehabil. 2014;41:836-42.

25. Rossetti LM, Rossetti PH, Conti PC, de Araujo Cdos R. Association between sleep bruxism and temporomandibular disorders: a polysomnographic pilot study. Cranio. 2008;26:16-24.

\section{Acknowledgments}

The authors thank the Coordination for Improvement of Higher Education Personnel (CAPES) for the financial support.

\section{Conflicts of Interest}

None to declare. 